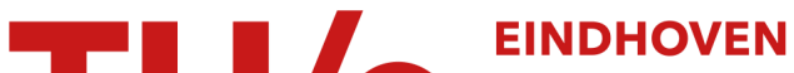 UNIVERSITY OF TECHNOLOGY
}

\section{Orientational mobility and relaxation spectra of dendrimers : theory and computer simulation}

\section{Citation for published version (APA):}

Markelov, D. A., Lyulin, S. V., Gotlib, Y. Y., Lyulin, A. V., Matveev, V. V., Lahderanta, E., \& Darinskii, A. A. (2009). Orientational mobility and relaxation spectra of dendrimers : theory and computer simulation. Journal of Chemical Physics, 130(4), 044907-1/9. [044907]. https://doi.org/10.1063/1.3063116

DOI:

10.1063/1.3063116

Document status and date:

Published: 01/01/2009

\section{Document Version:}

Publisher's PDF, also known as Version of Record (includes final page, issue and volume numbers)

\section{Please check the document version of this publication:}

- A submitted manuscript is the version of the article upon submission and before peer-review. There can be important differences between the submitted version and the official published version of record. People interested in the research are advised to contact the author for the final version of the publication, or visit the $\mathrm{DOI}$ to the publisher's website.

- The final author version and the galley proof are versions of the publication after peer review.

- The final published version features the final layout of the paper including the volume, issue and page numbers.

Link to publication

\section{General rights}

Copyright and moral rights for the publications made accessible in the public portal are retained by the authors and/or other copyright owners and it is a condition of accessing publications that users recognise and abide by the legal requirements associated with these rights.

- Users may download and print one copy of any publication from the public portal for the purpose of private study or research.

- You may not further distribute the material or use it for any profit-making activity or commercial gain

- You may freely distribute the URL identifying the publication in the public portal.

If the publication is distributed under the terms of Article $25 \mathrm{fa}$ of the Dutch Copyright Act, indicated by the "Taverne" license above, please follow below link for the End User Agreement:

www.tue.nl/taverne

Take down policy

If you believe that this document breaches copyright please contact us at:

openaccess@tue.nl

providing details and we will investigate your claim. 


\title{
Orientational mobility and relaxation spectra of dendrimers: Theory and computer simulation
}

\author{
Denis A. Markelov, ${ }^{1,2,3, a)}$ Sergey V. Lyulin, ${ }^{2}$ Yuli Y. Gotlib, ${ }^{2}$ Alexey V. Lyulin, ${ }^{4}$ \\ Vladimir V. Matveev, ${ }^{3}$ Erkki Lahderanta, ${ }^{1}$ and Anatolij A. Darinskii ${ }^{2}$ \\ ${ }_{1}^{1}$ Laboratory of Physics, Lappeenranta University of Technology, P.O. Box 20, 53851 Lappeenranta, Finland \\ ${ }^{2}$ Institute of Macromolecular Compounds, Russian Academy of Sciences, Bolshoi Prospect 31, V.O., \\ St. Petersburg 199004 Russia \\ ${ }^{3}$ Faculty of Physics, St. Petersburg State University, Ulyanovskaya Str. 1, Petrodvorets, St. Petersburg \\ 198504 Russia \\ ${ }^{4}$ Group Polymer Physics, Eindhoven Polymer Laboratories, Technische Universiteit Eindhoven, \\ P.O. Box 5135600 MB Eindhoven, The Netherlands and Dutch Polymer Institute, P.O. Box 9025600 AX \\ Eindhoven, The Netherlands
}

(Received 25 September 2008; accepted 3 December 2008; published online 29 January 2009)

\begin{abstract}
The developed theory of the orientational mobility of individual segments of a perfectly branched dendrimer is used to calculate the relaxation spectrum of a dendrimer. Frequency dependences of NMR relaxation $1 / T_{1}$ and of the nuclear Overhauser effect have been theoretically calculated from the Brownian dynamics simulation data. The dendrimer segmental orientational mobility is governed by three main relaxation processes: (i) the rotation of the dendrimer as a whole, (ii) the rotation of the dendrimer's branch originated from a given segment, and (iii) the local reorientation of the segment. The internal orientational mobility of an individual dendrimer segment depends only on the topological distance between this segment and the terminal shell of the dendrimer. Characteristic relaxation times of all processes and their contributions to the segmental mobility have been calculated. The influence of the number of generations and the number of the generation shell on the relaxation times has been studied. The correlation between the characteristic times and the calculated relaxation spectrum of the dendrimer has been established. () 2009 American Institute of Physics. [DOI: 10.1063/1.3063116]
\end{abstract}

\section{INTRODUCTION}

Dendrimers represent a new class of polymers characterized by regular architecture and nanosizes. ${ }^{1}$ Due to the specific topology ${ }^{2}$ dendrimers possess a number of unique properties which can define their potential applications in chemistry, biology, and medicine.

Most of experimental research of dendrimers is devoted to the studies of equilibrium properties (see, for example, Refs. 1-8). At the same time their relaxation properties, which are very important for many practical applications, are poorly understood. ${ }^{9-13}$ Computer simulation ${ }^{14-22}$ is one of the perspective methods to study the dendrimer relaxation properties. Obtained simulation results correlate well with the existing experimental data on large-scale relaxation processes, such as diffusion ${ }^{14}$ and intrinsic viscosity. ${ }^{15}$ However, the computational study of the local segmental mobility of a dendrimer faces difficulties due to the superposition of many relaxation processes and the absence of a theoretical approach for the interpretation of experimental data and computer simulation results (see, e.g., Refs. 14 and 19-22 where it is shown that the behavior of the orientational ACF $P_{1}$ for dendrimer segments

\footnotetext{
${ }^{a)}$ Author to whom correspondence should be addressed. Electronic mail: markeloved@gmail.com.
}

$$
P_{1}(t, i)=\left\langle\vec{b}_{i}(t) \vec{b}_{i}(0)\right\rangle
$$

is determined by several well-separated relaxation processes and $\vec{b}_{i}(t)$ is a unit vector directed along the $i$ th segment).

The local mobility of dendrimer segments is studied analytically using simple viscoelastic model of Gaussian subchains (segments). ${ }^{23-32}$ However, hydrodynamic and excluded-volume interactions, which play a major role in defining the dynamic properties of dendrimers, are not taken into account in this model. Therefore, the question arises to which extent the conclusions and predictions of this analytical approach is correct, in particular, in describing the relaxation of the $P_{1}$ autocorrelation function (ACF). This function may be calculated directly based on the dielectric relaxation, polarized luminescence, and NMR data. However, a correct theoretical approach describing its relaxation does not exist.

These open questions define the goals of the present study, namely, to examine the predictions of the analytical theory ${ }^{32}$ by comparison with the Brownian dynamics (BD) simulations results, ${ }^{14}$ and to explain the peculiarities of the time dependence of the $P_{1}$ ACF simulated by $\mathrm{BD}$ and measured in NMR experiment. The rest of the paper is organized as follows. In Sec. II we briefly describe some results of the viscoelastic theory, ${ }^{23-32}$ which have been used in the present study to analyze the orientational mobility of an individual segment (InS) of the dendrimer. In Sec. III we analyze the time dependence of the simulated ${ }^{14} P_{1}$ ACF for an InS of the 
dendrimer with the help of the viscoelastic theory. ${ }^{32}$ In Sec. IV we use theoretical predictions ${ }^{32}$ to calculate characteristic relaxation times of $P_{1}$ ACF based on NMR data. The main conclusions are summarized in Sec. V.

\section{VISCOELASTIC MODEL OF A DENDRIMER}

The well-known viscoelastic model of a dendrimer is described in details in many studies. ${ }^{23-32}$ In this model every two consequent branching points are connected by one segment of the same mean-squared length $\left\langle l^{2}\right\rangle$, described by the elasticity coefficient $K .^{33}$ The viscous friction is supposed to be concentrated in branching points and is characterized by the scalar friction coefficient $\zeta$. The free draining model is considered, and the excluded volume and hydrodynamic interactions (HIs) are not taken into account.

The spectrum of the relaxation times of normal modes in framework of this dendrimer model consists of two parts: internal relaxation spectrum and pulsating spectrum. As was shown earlier, ${ }^{25,27,28}$ internal relaxation spectrum is not much influenced on by the size of a dendrimer. The relaxation times of the internal relaxation spectrum lie in some interval between $\tau_{\min }^{\mathrm{int}}$ and $\tau_{\max }^{\mathrm{int}}$. At the same time, the pulsating spectrum strongly depends on a dendrimer size. The relaxation times of the pulsating spectrum are determined by the size of subbranches and are equal for subbranches of the same size. For every segment the subbranch to which it belongs can be defined uniquely; subbranch represents the dendrimer part originated from this segment, Fig. $1 .^{28}$ The subbranch is characterized by the number $m=n-j$, where $n$ is the total number of the dendrimer generations and $j$ is the number of a generation shell to which the given segment belongs to. The smallest subbranch with $m=0$ is just a terminal segment, Fig. 1(d). The largest subbranch is characterized by the number $m=(n-1)$, and corresponds to a branch originated from the dendrimer core [Fig. 1(a)]. As was already shown, ${ }^{25,27,28}$ for $m>1$ the pulsating relaxation time of a three-functional dendrimer, $\tau_{m}^{p u l}$, is equal to

$$
\tau_{m}^{p u l} \approx 2^{m+2} \tau_{0} \quad(m=2,3, \ldots, n) .
$$

Here $\tau_{0}=\zeta / K$ is the characteristic rotational time of a single dendrimer segment with one fixed end. The pulsating relaxation times for subbranches with $m=0$ and 1 have been derived by Gotlib and Markelov ${ }^{28}$ as

$$
\begin{aligned}
& \tau_{0}^{p u l}=\tau_{0} \quad(m=0), \\
& \tau_{1}^{p u l}=(2+\sqrt{3}) \tau_{0} \quad(m=1) .
\end{aligned}
$$

The degree of degeneracy $D$ of relaxation times of both spectra for $n=3$ and $n=5$ dendrimer has been shown in Figs. 2(a) and 2(b), correspondingly. This degree of degeneracy shows how often each relaxation time is found in the dendrimer spectrum, i.e., $D$ is the number of normal modes with the same relaxation time. Here solid lines mark the relaxation times of a dendrimer, dashed lines label the borders of the internal spectrum, $\tau_{\mathrm{av}}^{\mathrm{int}}$ is the average time of the internal spectrum (this time is determined below).

In the viscoelastic model described above, every segment is flexible and represent normally several monomer
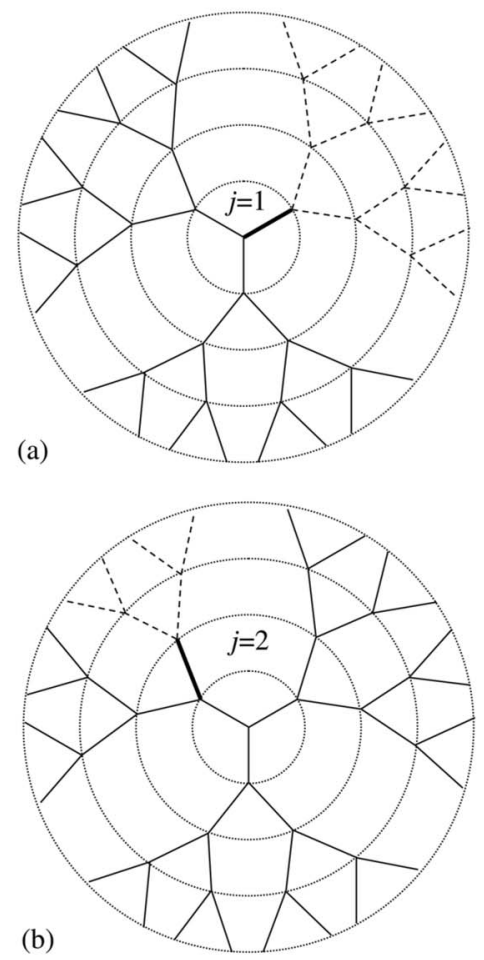

(b)
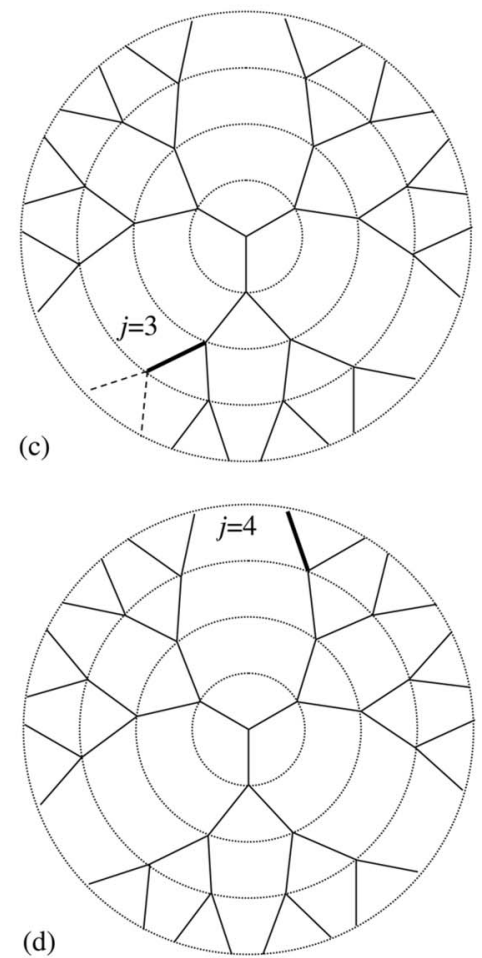

FIG. 1. The generation $n=4$ dendrimer. InSs are topologically located in an arbitrary generation shell of a dendrimer: (a) $j=1, m=3$; (b) $j=2, m=2$; (c) $j=3, m=1$, and (d) $j=4, m=0 . j$ is the number of a generation shell of an InS, $m$ is the number of generations of a marked subbranch. Dotted lines mark shells, the thick line highlights the InS, the dashed line indicates the marked subbranch.

units of a real polymer chain. However, a majority of known dendrimers have very rigid segments and short spacers between branching points. Gotlib and Neelov ${ }^{34}$ studied the relaxation spectrum of a free-draining dendrimer model where all segments are substituted by rigid rods (bead-rod model). 

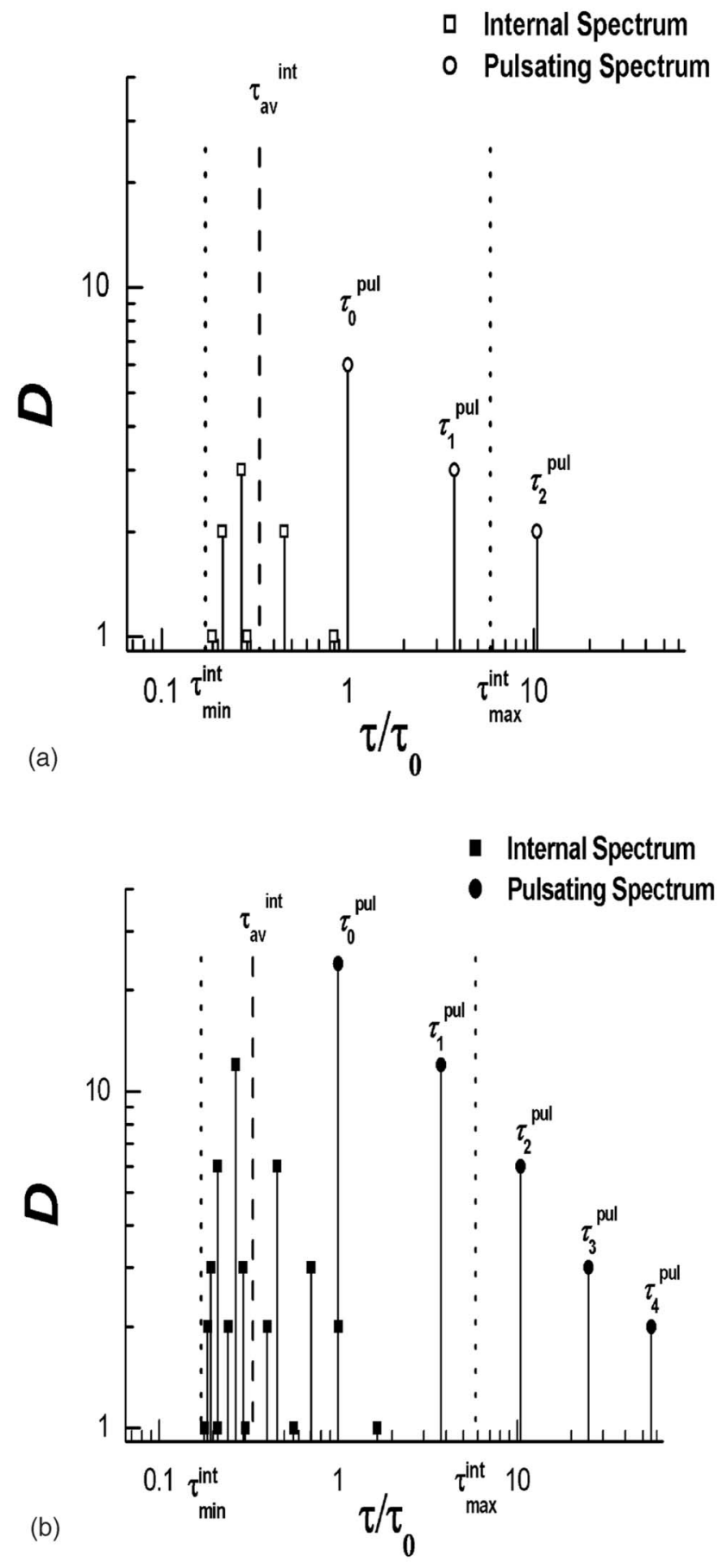

FIG. 2. The analytical theory predictions (Ref. 28) for the degree of degeneracy $D$ of both the internal spectrum and the pulsating spectrum for (a) $n$ $=3$ and (b) $n=5 . m$ is the number of generations of a marked subbranch, $n$ is the number of generations of a dendrimer, $\tau_{0}$ is the characteristic relaxation time of a single segment, $\tau_{m}^{\text {pul }}$ is the relaxation time of the pulsating spectrum, $\tau_{\min }^{\text {int }}$ and $\tau_{\max }^{\text {int }}$ are the limiting values for the relaxation times of the internal spectrum (dashed lines), and $\tau_{\mathrm{av}}^{\mathrm{int}}$ is the average relaxation time of the internal spectrum (dotted lines).

They showed that the relaxation spectra for both bead-rod and viscoelastic models coincide with each other if the length of a rod in the rigid model is equal to the meansquared length of a segment in the viscoelastic model.

For an InS belonging to the $j$ th generation shell the viscoelastic theory of a $n$-generations dendrimer ${ }^{32}$ gives the following approximate expression for the time dependence of the $P_{1}$ ACF:

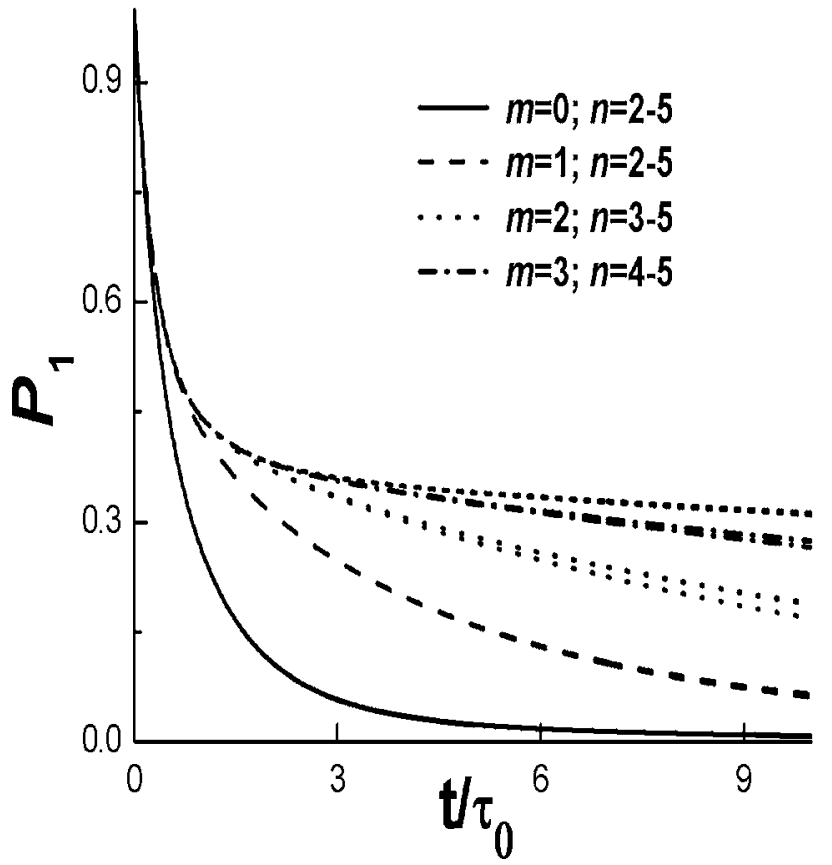

FIG. 3. The theoretical predictions (Ref. 32) for the time dependence of the $P_{1}$ ACF of InS of a $n$-generations dendrimer at different numbers of generations $m=n-j$ for a marked subbranch. $j$ is the number of a generation shell of the InS, $\tau_{0}$ is the characteristic relaxation time of a single segment.

$$
P_{1}(t, n, j)=A(n, j) \exp \left(-t / \tau_{n-j}^{\mathrm{pul}}\right)+B(n, j) \exp \left(-t / \tau_{\mathrm{av}}^{\mathrm{int}}\right),
$$

where $\tau_{n-j}^{\mathrm{pul}}$ is the pulsating relaxation time of the subbranch that originates from this segment; $\tau_{\mathrm{av}}^{\mathrm{int}}$ is the characteristic relaxation time of the internal spectrum; the expression for $\tau_{\mathrm{av}}^{\mathrm{int}}$ is given below [Eq. (7)]. The contributions $A$ and $B$ of relaxation times must satisfy the normalization condition:

$$
A+B=1 \text {. }
$$

An initial slope of the $P_{1}(t, n, j)$ ACF is determined only by the internal spectrum and does not practically depend on $n$ and $j$ because $\tau_{\mathrm{av}}^{\mathrm{int}} \ll \tau_{n-j}^{\text {pul }}$ (Fig. 3). The relationship between the characteristic relaxation time $\tau_{\mathrm{av}}^{\mathrm{int}}$ and the limiting values of the internal spectrum of the dendrimer has been also obtained as ${ }^{32}$

$$
\frac{1}{\tau_{\mathrm{av}}^{\mathrm{int}}} \approx \frac{1}{2}\left(\frac{1}{\tau_{\min }^{\mathrm{int}}}+\frac{1}{\tau_{\max }^{\mathrm{int}}}\right) .
$$

At large times, the relaxation of $P_{1}(t, n, j)$ is determined, obviously, by the larger relaxation time $\tau_{n-j}^{\text {pul }}$. For the $n$-generation dendrimer $\tau_{n-j}^{\text {pul }}$ is equal to the maximum pulsating time of a subbranch with the number of generations $m$ $=n-j$, see Eq. (2).

The ratio $A / B$ depends only on the number $m$ of generations for the corresponding subbranch and decreases when $m$ increases, Fig. 4. Therefore, we can rewrite Eq. (5) as

$$
P_{1}(t, n, m)=A_{m} \exp \left[-t / \tau_{m}^{\mathrm{pul}}\right]+B_{m} \exp \left[-t / \tau_{\mathrm{av}}^{\mathrm{int}}\right] .
$$

As follows from Eq. (8):

$$
P_{1}\left(t, n^{\prime}, m\right)=P_{1}\left(t, n^{\prime \prime}, m\right), \quad\left(m=n^{\prime}-j^{\prime}=n^{\prime \prime}-j^{\prime \prime}\right),
$$

where $n^{\prime}$ and $n^{\prime \prime}$ are the numbers of dendrimer generations, $j^{\prime}$ and $j^{\prime \prime}$ are the numbers of generation shells for the selected 


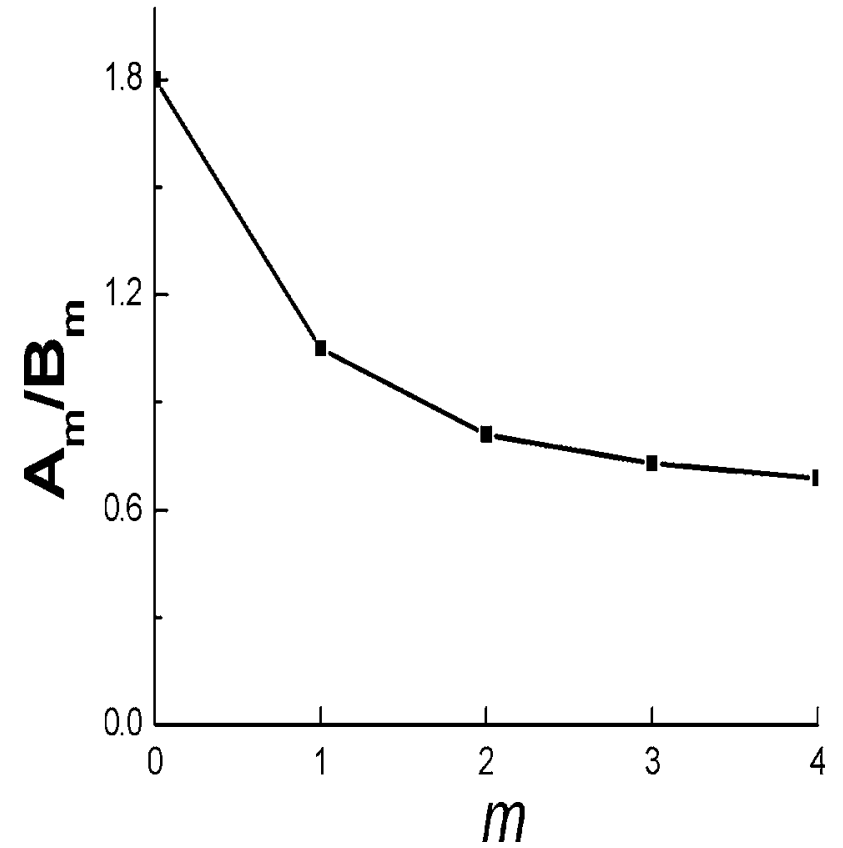

FIG. 4. The theoretical predictions (Ref. 32) for the ratio $A_{m} / B_{m}$ of pulsating and internal characteristic times as a function of the number of generations $m$ of a marked subbranch.

segments. In other words, the expressions for $P_{1}$ ACFs are the same for different segments, if these segments are characterized by the same $m .^{32}$ Thus, the orientational mobility of InS of a dendrimer is determined only by the topological distance of this segment from the terminal shell, i.e., by the size of the corresponding subbranch instead of the size of the dendrimer. Equations (8) and (9) will be used below to analyze the time dependence of $P_{1}$ ACF obtained earlier in the BD simulation study. ${ }^{14}$

\section{BROWNIAN DYNAMICS SIMULATION OF THE LOCAL ORIENTATIONAL MOBILITY IN DENDRIMERS}

The time behavior of the $P_{1}$ ACF has been simulated ${ }^{14}$ for a single dendrimer up to $n=5$ generations for two models, with and without HIs. The dendrimer was represented as a system of beads with friction coefficient $\zeta$ connected by rigid rods of length $l$. Excluded-volume interactions were defined by the repulsive part of the Lennard-Jones potential $U^{\mathrm{LJ}}$ corresponding to the athermal solvent, with the cutoff distance $r_{\text {cut }}=2.5 \sigma$ and parameters $\sigma=0.8 l$ and $\varepsilon_{\mathrm{LJ}}=0.3 k_{b} T,{ }^{35}$

$$
U^{\mathrm{LJ}}\left(r_{i j}\right)=4 \varepsilon_{\mathrm{LJ}}\left(\frac{\sigma}{r_{i j}}\right)^{12}-4 \varepsilon_{\mathrm{LJ}}\left(\frac{\sigma}{r_{\mathrm{cut}}}\right)^{12} .
$$

As was shown in Ref. 14 for both models Eq. (9) does not work properly to describe the BD results, and the time dependences of $P_{1} \mathrm{ACF}$ can be different for the same values of $m$. We suppose that this discrepancy could be related to the rotation of the dendrimer as a whole because this type of motion is not taken into account in the theoretical treatment, Eq. (9).
TABLE I. The BD-simulated time $\tau_{\text {rot }}$ of a rotation of a dendrimer as a whole for two models, with and without HIs (Ref. 14). $n$ is the number of generations of a dendrimer, $\tau_{0}$ is the characteristic relaxation time of a single segment.

\begin{tabular}{ccc}
\hline \hline & \multicolumn{2}{c}{$\left(\tau_{\text {rot }} / \tau_{0}\right)$} \\
\cline { 2 - 3 }$n$ & Without $\mathrm{HI}$ & With HI \\
\hline 2 & 4 & 2.9 \\
3 & 10 & 7.5 \\
4 & 22.5 & 17 \\
5 & 48.6 & 34 \\
\hline \hline
\end{tabular}

\section{A. Contribution of a dendrimer rotation as a whole to segmental relaxation.}

The rotational mobility of a dendrimer as a whole is characterized by the relaxation times $\tau_{\text {rot }}$ of the correlation function $C_{\text {rot }}(t)$

$$
C_{\text {rot }}(t)=\left\langle\boldsymbol{e}_{n}(0) \boldsymbol{e}_{n}(t)\right\rangle .
$$

Here $\vec{e}_{n}=\vec{Q}_{n} /\left|\vec{Q}_{n}\right|$ is a unit vector that is collinear with the radius vector $\vec{Q}_{n}$ directed from the core to a terminal segment,

$$
\vec{Q}_{n}=\vec{r}_{t}-\vec{r}_{0},
$$

where $\vec{r}_{t}$ represents the location of an arbitrary $t$ th terminal segment for a $n$-generation dendrimer, and the vector $\vec{r}_{0}$ represents the position of a dendrimer core. Averaging in Eq. (11) is performed over all terminal groups and over time. In the Table I we present relaxation times $\tau_{\text {rot }}$ calculated in BD simulations for both models, with and without HI. ${ }^{14}$ The value of $\tau_{\text {rot }}$ increases with increasing the number of generations $n$.

The contribution of the rotation of the dendrimer as a whole may be taken into account by adding a new mode in Eq. (8) (this approach is similar to that used earlier for a single polymer chain ${ }^{36}$ ),

$$
\begin{aligned}
P_{1}(t, n, m)= & A_{m} \exp \left[-t / \tau_{m}^{\mathrm{pul}}\right]+B_{m} \exp \left[-t / \tau_{\mathrm{av}}^{\mathrm{int}}\right] \\
& +C_{m} \exp \left[-t / \tau_{\mathrm{rot}}(n)\right] .
\end{aligned}
$$

Now, the sum of all three contributions of the characteristic relaxation processes has to satisfy a new normalization condition:

$$
A_{m}+B_{m}+C_{m}=1
$$

As follows from Eq. (14) both $A_{m}, B_{m}$, and $C_{m}$ depend on the $m=n-j$ only. The value of $C_{m}$ can be calculated from the simulation data for segments of dendrimers of different generation numbers (for example, $n^{\prime}$ and $n^{\prime \prime}$ ), but with the same number of subbranch generations $m=n^{\prime}-j^{\prime}=n^{\prime \prime}-j^{\prime \prime}$ :

$$
\begin{aligned}
P_{1}\left(t, n^{\prime}, m\right)-P_{1}\left(t, n^{\prime \prime}, m\right)= & C_{m}\left(\exp \left[-t / \tau_{\text {rot }}\left(n^{\prime \prime}\right)\right]\right. \\
& \left.-\exp \left[-t / \tau_{\text {rot }}\left(n^{\prime}\right)\right]\right) .
\end{aligned}
$$

The calculated values $C_{m}(m=0,1,2)$ for different segments are close to each other, and are presented in Table II for both simulated models. These results confirm our hypothesis that the difference in the time dependence of $P_{1}$ 
TABLE II. Characteristic BD-simulated relaxation times of pulsating and internal spectra and their contributions to $P_{1}$ ACF relaxation for two models with and without HIs (Ref. 14). $\tau_{m}^{\text {pul }}$ is the characteristic pulsating time, $\tau_{\mathrm{av}}^{\mathrm{int}}$ is the characteristic time of the internal spectrum, $C_{m}$ is the contribution of the rotation of a dendrimer as a whole, $A_{m}$ is the contribution of a motion with $\tau_{m}^{\text {pul }}, B_{m}$ is the contribution of a motion with $\tau_{\mathrm{av}}^{\mathrm{int}}, m=n-j$ is the number of generations of a marked subbranch, $j$ is the number of a generation shell of the InS, $n$ is the number of generations of a dendrimer, and $\tau_{0}$ is the characteristic relaxation time of a single segment.

\begin{tabular}{cccccc}
\hline \hline \multicolumn{5}{c}{ Model without HIs } \\
\hline$m$ & $C_{m}$ & $A_{m}$ & $\tau_{m}^{\mathrm{pul}} / \tau_{0}$ & $B_{m}$ & $\tau_{\mathrm{av}}^{\text {int }} / \tau_{0}$ \\
0 & $0.06 \pm 0.02$ & $0.50 \pm 0.02$ & $1.27 \pm 0.05$ & $0.44 \pm 0.02$ & $0.17 \pm 0.05$ \\
1 & $0.26 \pm 0.01$ & $0.47 \pm 0.01$ & $3.9 \pm 0.2$ & $0.27 \pm 0.01$ & $0.17 \pm 0.02$ \\
2 & $0.36 \pm 0.01$ & $0.38 \pm 0.01$ & $26 \pm 1$ & $0.26 \pm 0.01$ & $0.20 \pm 0.02$ \\
& \multicolumn{5}{c}{ Model with HIs } \\
0 & $0.06 \pm 0.02$ & $0.59 \pm 0.02$ & $1.09 \pm 0.05$ & $0.35 \pm 0.02$ & $0.20 \pm 0.05$ \\
1 & $0.24 \pm 0.01$ & $0.47 \pm 0.01$ & $3.6 \pm 0.2$ & $0.29 \pm 0.01$ & $0.19 \pm 0.02$ \\
2 & $0.41 \pm 0.01$ & $0.33 \pm 0.01$ & $11.9 \pm 0.5$ & $0.26 \pm 0.01$ & $0.21 \pm 0.02$ \\
\hline \hline
\end{tabular}

$(t, n, m)$ for the same $m$ is determined mainly by the difference in rotation relaxation times of a dendrimer as a whole.

\section{B. Calculation of parameters of dendrimer local motions in $P_{1}$ relaxation}

Having the contributions to $P_{1}$ ACF of the dendrimer rotation as a whole, we introduce another ACF, $\widetilde{P}_{1}$, which depends only on the local motions of a segment within a dendrimer. It allows us to study the remaining parameters of $P_{1}$ ACF. To calculate the parameters in Eq. (13), the contribution $P_{1}^{\mathrm{rot}}(t, n, m)=C_{m} \exp \left[-t / \tau_{\mathrm{rot}}(n)\right]$ of the rotation of a dendrimer as a whole is subtracted from $P_{1}(t, n, m)$,

$$
\begin{aligned}
\widetilde{P}_{1}(t, m) & =P_{1}\left(t, n^{\prime}, m\right)-C_{m} \exp \left[-t / \tau_{\text {rot }}\left(n^{\prime}\right)\right] \\
& =P_{1}\left(t, n^{\prime \prime}, m\right)-C_{m} \exp \left[-t / \tau_{\text {rot }}\left(n^{\prime \prime}\right)\right] .
\end{aligned}
$$

Calculated $\widetilde{P}_{1}$ ACF is determined only by the internal orientational mobility of dendrimer segments and depends only on the number of generations $m$ in the marked subbranch, see Eq. (8). In Fig. 5 the time dependence of $\widetilde{P}_{1}$ ACF for segments with the number $m$ of subbranch generations is shown. All $\widetilde{P}_{1}(t, m)$ ACFs are well fitted by Eq. (8). Thus, relaxation times $\tau_{m}^{\text {pul }}, \tau_{\mathrm{av}}^{\text {int }}$ and their contributions $A_{m}, B_{m}$ that determine the BD simulation time dependence of $P_{1}$ ACF can be defined. In Table II the calculated values of $A_{m}, B_{m}, \tau_{m}^{\mathrm{pul}}$, and $\tau_{\mathrm{av}}^{\mathrm{int}}$ are shown for models with and without HI.

\section{Analysis of obtained parameters $A_{m}, B_{m}, C_{m}, \tau_{m}^{\mathrm{pul}}$, $\tau_{\mathrm{av}}^{\mathrm{jin}}$, and $\tau_{\text {rot }}$}

In this section the relaxation times of $P_{1} \mathrm{ACF}$ for the viscoelastic model and for the simulated bead-rod model are analyzed in detail. In BD simulations the characteristic time $\tau_{\mathrm{av}}^{\mathrm{int}} \approx 0,2 \tau_{0}$ is the same for both models, with and without $\mathrm{HI}$, and does not depend on the dendrimer generation number $n$ and an InS topological location. This result agrees with the theoretical predictions for viscoelastic model, ${ }^{32}$ i.e., this time belongs to the internal spectrum and corresponds to the local reorientational motions of $\mathrm{InS}$ for dendrimers with slow mobility of the terminal shell. The characteristic time $\tau_{m}^{\text {pul }}$ is related to the pulsations of the corresponding subbranch and increases with increasing $m$. Indeed, the BD simulation results show this increase. For the model without HI, this effect is larger than for the model with HI, Fig. 6. In Fig. 6, the relaxation times $\tau_{m}^{\text {pul }}$ and $\tau_{\mathrm{av}}^{\text {int }}$ are shown for both simulated ${ }^{14}$ models, with and without HI, and for the viscoelastic model. ${ }^{32}$ Note that pulsating relaxation times of the BD model with $\mathrm{HI}$ are close to the values of the pulsating times for the viscoelastic model (see Fig. 6). This fact shows the mutual compensation of the excluded volume and HI interactions that are not taken into account in the viscoelastic model. Thus, we conclude here that the simulated time dependence of the $P_{1}$ ACFs is well described by Eq. (13) and is defined completely by three main relaxation processes:

- rotation of the dendrimer as a whole,

- turns of the marked subbranch characterized by the maximal pulsating relaxation time, and

- local reorientational motions of an InS characterized by the average characteristic time of the internal spectrum.

The contribution of each process depends on the number $m$ of generations in the marked subbranch (Table II). However, the time dependence of $P_{1} \mathrm{ACF}$ is determined not only by $m$ but also by $n$ because the rotation time of a dendrimer as a whole is determined only by its size, i.e., by the number $n$ of generations for a chosen model. For terminal InS ( $m$ =0) $P_{1}$ does not depend on $n$ because the contribution of the normal mode corresponding to $\tau_{\text {rot }}$ is negligible in this case.

The conclusions of the developed theory correlate well with the results of the molecular-dynamics simulations of dendrimers by Karatasos et al. ${ }^{19}$ In Ref. 19 AB2 dendrimers of generations $n=4-7$ have been simulated in a dilute solution with explicit solvent molecules. To study the relaxation processes which determine the orientational mobility of InS, the authors calculated the distribution of relaxation times, $F(\ln (\tau))$. This function is connected to $P_{1}(t)$ as $P_{1}(t)$ $=\int_{-\infty}^{\infty} F(\ln (\tau)) e^{-t / \tau} d \ln (\tau) .{ }^{19}$ Maxima of $F(\ln (\tau))$ may be recognized as corresponding characteristic times of $P_{1}$ relaxation, if these times are well separated from each other. Otherwise, contributions of different relaxation times merge into one maximum. Thus, for rather short subbranches $(m=0$ and 

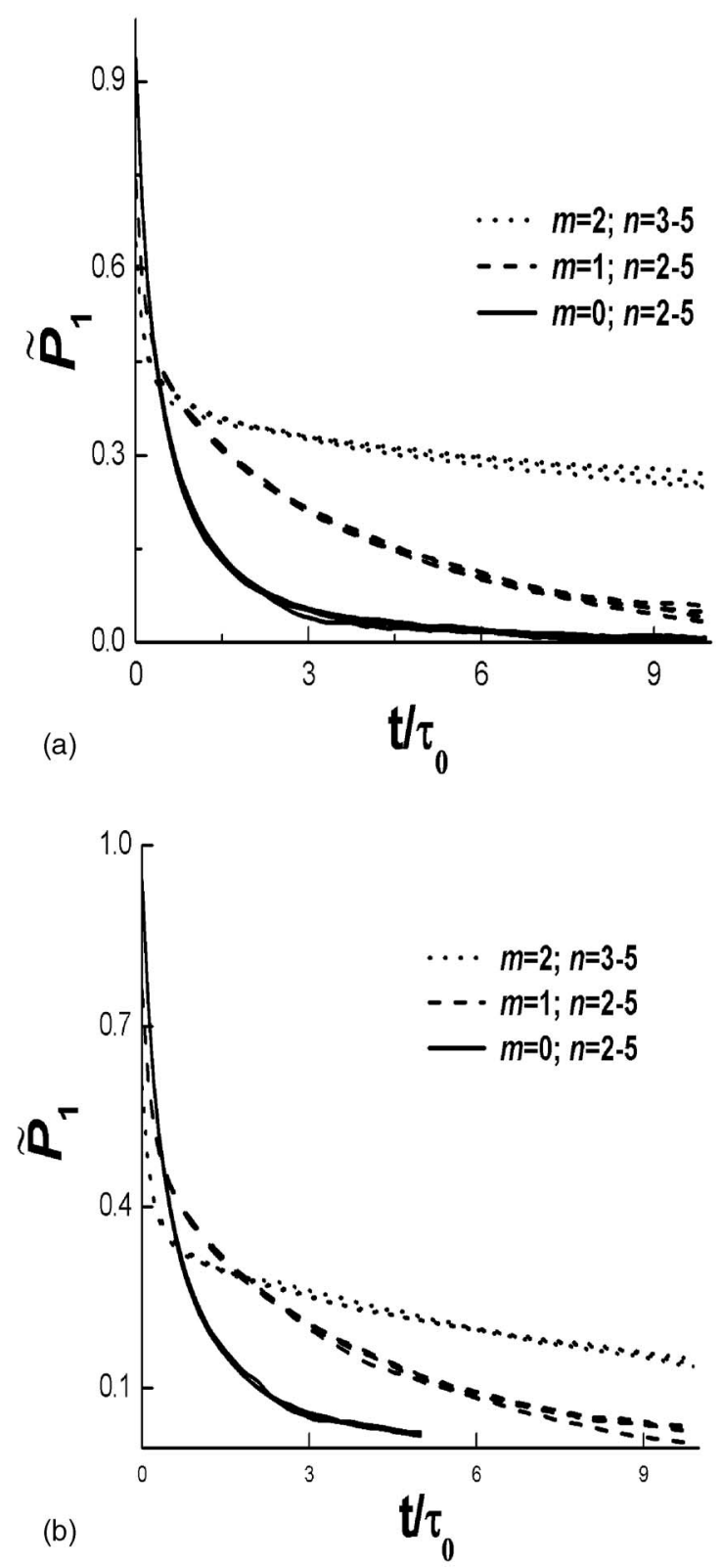

FIG. 5. The time dependence of $\widetilde{P}_{1}(t, m)$ ACF. $m=n-j$ is the number of generations of a marked subbranch, $j$ is the number of a generation shell of the InS, $n$ is the number of generations of a dendrimer, and $\tau_{0}$ is the characteristic relaxation time of a single segment. Shown are the results of the BD simulations for models (b) with and (a) without HIs (Ref. 14).

1) function $F(\ln (\tau))$ is characterized in Ref. 19 by one maximum due to a small contribution of the dendrimer rotation as a whole in this case. This maximum corresponds to a joint contribution of the internal spectrum and pulsating processes because the characteristic time $\tau_{\mathrm{av}}^{\mathrm{int}}$ is close to the value of $\tau_{m}^{\text {pul. }}$. However, for larger dendrimers $(n=4-6$ and $m=2-n)$ two relaxation processes are split. ${ }^{19}$ The characteristic time of the faster process does not depend practically on $n$ and $m$. The contribution of this process decreases with increasing $m$. This process corresponds to the contribution of the internal spectrum in $P_{1} \mathrm{ACF}$ (see discussion at the beginning of this section). The characteristic time of the second process increases with $m$, and for $m=n-2$ becomes close to $\tau_{\text {rot }}{ }^{19} \mathrm{We}$ suppose that observed process is the superposition of pulsa-

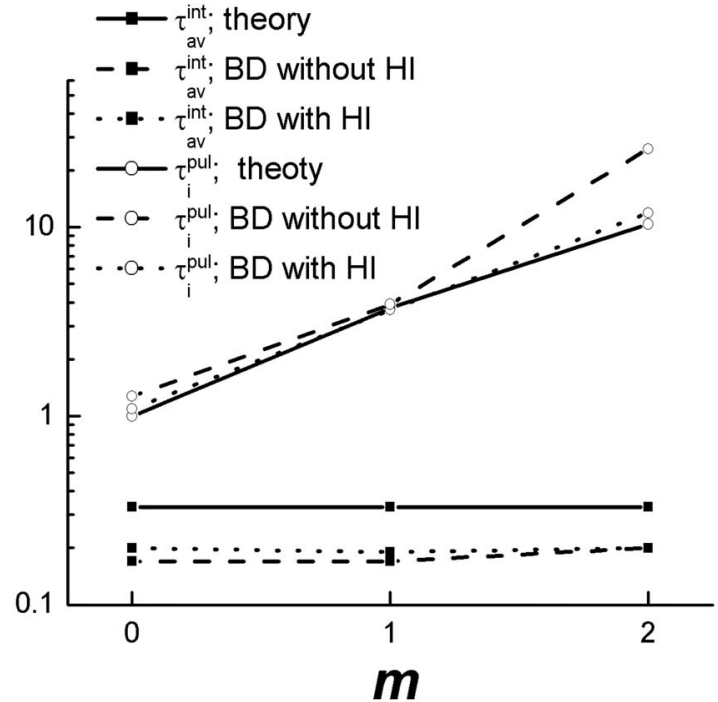

FIG. 6. Characteristic relaxation times ( $\tau_{m}^{\mathrm{pul}}$ and $\tau_{\mathrm{av}}^{\mathrm{int}}$ ) for the relaxation of $P_{1}$ ACF. $m=n-j$ is the number of generations of a marked subbranch, $j$ is the number of a generation shell of the InS, $n$ is the number of generations of a dendrimer, and $\tau_{0}$ is the characteristic relaxation time of a single segment. Shown are the results of the analytical theory (Ref. 32) and the BD simulations (Ref. 14).

tion $\left(\tau_{m}^{\text {pul }}\right)$ and the dendrimer rotation as a whole $\left(\tau_{\text {rot }}\right)$ because the difference between corresponding relaxation times $\left(\tau_{m}^{\mathrm{pul}}\right.$ and $\left.\tau_{\text {rot }}\right)$ is not enough to separate these processes. Our hypothesis is verified by the shape of the $F(\ln (\tau))$ for $n=7$ generation dendrimer. ${ }^{19}$ In this case $(n=7$ and $m=2-4)$, three characteristic times are clearly recognized. The processes with faster and moderate characteristic times have the similar behavior as for the smaller system with only two maxima $(n<7)$. As was shown in Ref. 19 , the third process has a maximal relaxation time and corresponds to the dendrimer rotation as a whole. The characteristic times of this process are practically the same for $m=2-4$.

Thus, the developed analytical theory of local orientational mobility correctly describes time dependence of $P_{1}$ ACF for separate segments produced by computer simulations. ${ }^{14}$ The studies of the orientational mobility of InS in different shells allow us to calculate the pulsating spectrum $\tau_{m}^{\text {pul }}$ [see Eqs. (2)-(4)], the average time $\tau_{\mathrm{av}}^{\text {int }}$ of the internal spectrum, and finally, the full relaxation spectrum of a dendrimer, Fig. 2. The relaxation spectrum can be further used for description and investigation of various relaxation processes in a dendrimer that are observed in computer simulations and in some physical experiments such as dielectric and mechanical relaxation, and NMR. Moreover, the comparison of the viscoelastic theory with BD data allows us to improve analytical description of $P_{1}$ relaxation, namely, that $P_{1}$ ACF should additionally include the rotation of a dendrimer as a whole.

\section{MANIFESTATIONS OF THE ORIENTATIONAL MOBILITY OF DENDRIMER SEGMENTS IN NMR}

The orientational mobility of a dendrimer segment can be displayed in the reorientation of an internuclear vector, which is observed in NMR experiments. In this section we will use theoretical results of the present study to analyze 
(a)

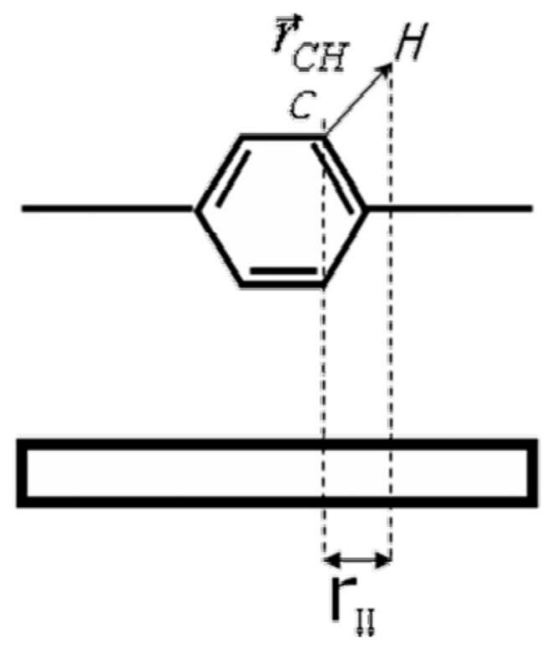

FIG. 7. (a) A dendrimer segment with benzene ring (Refs. 37-39) and (b) a bead-rod model of its segment which is used in this study. $\vec{r}_{\mathrm{CH}}$ is the internuclear vector of a $\mathrm{C}-\mathrm{H}$ bond, $\vec{r}_{\mathrm{II}}$ is the longitudinal component of the internuclear vector of a $\mathrm{C}-\mathrm{H}$ bond.

NMR data. In the developed theory only the longitudinal components of the internuclear vector have been investigated, Eq. (1). Such situation is realized, for example, in polyphenylene dendrimers ${ }^{37-39}$ where $\mathrm{C}-\mathrm{H}$ bonds possess the longitudinal component of the internuclear vector, Fig. 7.

The information about the local orientational mobility of a dendrimer can be obtained from the NMR relaxation of ${ }^{13} \mathrm{C}$ nuclei or from the nuclear Overhauser effect (NOE). The inverse time of the NMR relaxation, $1 / T_{1 \mathrm{C}}$, and the value of NOE can be represented as (see, for example, Refs. 40-42):

$$
\begin{aligned}
1 / T_{1 \mathrm{C}}(\omega, n, m)= & \frac{\gamma_{C}^{2} \gamma_{H}^{2} \hbar^{2}}{10 r_{\mathrm{II}}^{6}}[6 J(4.97 \omega, n, m) \\
& +J(2.97 \omega, n, m)+3 J(\omega, n, m)],
\end{aligned}
$$

$\operatorname{NOE}(\omega, n, m)$

$$
=1+3.976 \frac{6 J(4.97 \omega, n, m)-J(2.97 \omega, n, m)}{6 J(4.97 \omega, n, m)+J(2.97 \omega, n, m)+3 J(\omega, n, m)},
$$

where $\gamma_{\mathrm{H}}$ and $\gamma_{\mathrm{C}}$ are the gyromagnetic ratios for nuclei ${ }^{1} \mathrm{H}$ and ${ }^{13} \mathrm{C}$, correspondingly; $r_{\mathrm{II}}$ is the projection of the internuclear vector on the axis directed along the InS, and $J(\omega, n, m)$ is the spectral density function, which is calculated from the real part of the Fourier transform of the time dependence of $P_{2}(t, n, m)$ ACF. The orientational ACF $P_{2}$ is defined as

$$
P_{2}(t)=\frac{3}{2}\left(\left\langle\left(\vec{b}_{i}(t) \vec{b}_{i}(0)\right)^{2}\right\rangle-\frac{1}{3}\right),
$$

where $\vec{b}_{i}(t)$ is a unit vector directed along the $i$ th segment.

We do not directly calculate $P_{2} \mathrm{ACF}$, but use the following approach instead. In Ref. 43 the following relation between $P_{1}$ and $P_{2}$ for rigid segments has been obtained:

$$
P_{2}(t)=\left(P_{1}(t)\right)^{3} \text {. }
$$

BD simulations results ${ }^{14}$ for both models (with and without HI) demonstrate that Eq. (20) is valid also for a dendrimer model with rigid segments. Thus, using our theoretical pre-

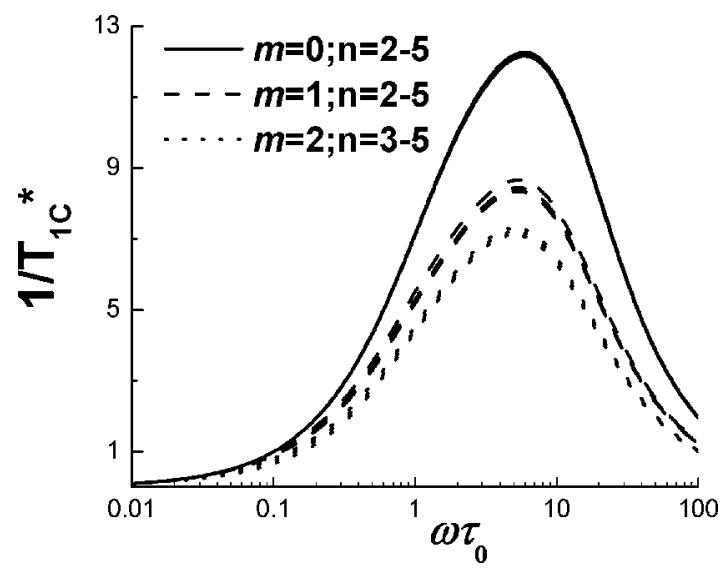

FIG. 8. The frequency dependence of the normalized inverse time of the NMR relaxation, $1 / T_{1 \mathrm{C}}^{*}(\omega, n, m)=\left(10 r_{\mathrm{II}}^{6} / \gamma_{\mathrm{C}}^{2} \gamma_{\mathrm{H}}^{2} \hbar^{2}\right) \omega / T_{1 \mathrm{C}}(\omega, n, m)$, for InSs of a dendrimer. $m$ is the number of generations of a marked subbranch, $n$ is the number of generations of a dendrimer, $\tau_{0}$ is the characteristic relaxation time of a single segment.

dictions, Eq. (13), we can obtain the relationship between $1 / T_{1}$ (or NOE) and the $P_{1}$ relaxation time. Using Eqs. (13) and (20), the expression for $P_{2}$ ACF can be written as

$$
\begin{aligned}
P_{2}(t, n, m)= & \sum_{l_{1}=1}^{3} \sum_{l_{2}=1}^{3} \sum_{l_{3}=1}^{3}\left(g_{l_{1}}(m) g_{l_{2}}(m) g_{l_{3}}(m)\right. \\
& \left.\times \exp \left[-\frac{t}{\tau_{l_{1} l_{2} l_{3}}}\right]\right),
\end{aligned}
$$

where $g_{1}(m)=A_{m}, g_{2}(m)=B_{m}$, and $g_{3}(m)=C_{m}$, (see Table II); $\tau_{l_{1} l_{2} l_{3}}$ are the characteristic relaxation times of $P_{2} \mathrm{ACF}$,

$$
\tau_{l_{1} l_{2} l_{3}}=\left(\frac{1}{\tau_{l_{1}}}+\frac{1}{\tau_{l_{2}}}+\frac{1}{\tau_{l_{3}}}\right)^{-1} .
$$

Here, $\tau_{1}=\tau_{m}^{\mathrm{pul}}, \tau_{2}=\tau_{\mathrm{av}}^{\mathrm{int}}$, and $\tau_{3}=\tau_{\mathrm{rot}}(n)$.

Therefore, we have

$$
J(\omega, n, m)=\sum_{l_{1}=1}^{3} \sum_{l_{2}=1}^{3} \sum_{l_{3}=1}^{3} \frac{g_{l_{1}}(m) g_{l_{2}}(m) g_{l_{3}}(m)}{1+\left(\tau_{l_{1} l_{2} l_{3}} \omega\right)^{2}} .
$$

We have calculated the NMR relaxation for the more realistic model with HI. Using Eqs. (17) and (23), and the calculated values of $\tau_{m}^{\text {pul }}, \tau_{\mathrm{av}}^{\mathrm{int}}, \tau_{\text {rot }},(n), A_{m}, B_{m}$, and $C_{m}$ for the model with HI (see Tables I and II), we obtain the frequency dependence of the $1 / T_{1 C}^{*}(\omega, n, j)$

$$
1 / T_{1 \mathrm{C}}^{*}(\omega, n, m)=\left(\frac{10 r_{\mathrm{CH}}^{6}}{\gamma_{\mathrm{C}}^{2} \gamma_{\mathrm{H}}^{2} \hbar^{2}}\right) \frac{\omega}{T_{1 \mathrm{C}}(\omega, n, m)} .
$$

In Fig. 8, the frequency dependence of $1 / T_{1 C}^{*}$ is shown for dendrimers with different number of generations. The frequency $\omega_{\max }$ of the maximal value of the $1 / T_{1 C}^{*}$ does not depend practically on the size of a dendrimer and on the topological location of the InS in it. As was shown earlier, ${ }^{38}$ $\omega_{\max }$ relates to the characteristic time $\tau_{\mathrm{ch}}$ of $P_{2} \mathrm{ACF}$ as 


$$
\tau_{\mathrm{ch}}=\frac{1}{0.63 \omega_{\max }} .
$$

For the model with HI we obtain $\tau_{\mathrm{ch}} \approx \tau_{\mathrm{av}}^{\mathrm{int}} / 2 \approx 0.1 \tau_{0}$. These results can be explained by the fact that segmental motions with relaxation times $\tau_{122}$ and $\tau_{322}$ [see Eqs. (26) and (27)] give maximal contributions to relaxation of the $P_{2} \mathrm{ACF}$

$$
\begin{aligned}
& \tau_{122}=\left(\frac{1}{\tau_{m}^{\mathrm{pul}}}+\frac{1}{\tau_{\mathrm{av}}^{\mathrm{int}}}+\frac{1}{\tau_{\mathrm{av}}^{\mathrm{int}}}\right)^{-1}=\left(\frac{1}{\tau_{m}^{\mathrm{pul}}}+\frac{2}{\tau_{\mathrm{av}}^{\mathrm{int}}}\right)^{-1} \approx \tau_{\mathrm{av}}^{\mathrm{int}} / 2 \\
& \tau_{322}=\left(\frac{1}{\tau_{\mathrm{rot}}(n)}+\frac{2}{\tau_{\mathrm{av}}^{\mathrm{int}}}\right)^{-1} \approx \tau_{\mathrm{av}}^{\mathrm{int}} / 2
\end{aligned}
$$

Thus, the frequency dependence of $1 / T_{1 \mathrm{C}}^{*}(\omega, n, m)$ (Fig. 8) can be approximated as

$$
1 / T_{1 \mathrm{C}}^{*}(\omega, m) \approx \frac{L_{m} \omega}{1+\left(0.315 \tau_{\mathrm{av}}^{\mathrm{int}} \omega\right)^{2}} .
$$

Here $L_{m}$ is constant that depends only on $m$. Note that Eq. (28) can be used to test the developed theory and to calculate $\tau_{\mathrm{av}}^{\mathrm{int}}$ from the frequency dependence of $1 / T_{1 \mathrm{C}}^{*}$ obtained in a NMR experiment. Equation (28) is indirectly justified by the experimental investigation of NMR relaxation in DAB dendrimers (see Ref. 44). In Ref. 44 the temperature dependence of the NMR relaxation of $\mathrm{H}$ (Ref. 1) nuclei, $T_{1 \mathrm{H}}$, was studied. Their results ${ }^{44}$ clearly show that for different dendrimers $(n$ $=2,4,5)$ the positions of the $1 / T_{1 \mathrm{H}}$ maxima for all dendrimer segments coincide.

Similar to the $1 / T_{1 \mathrm{C}}^{*}$ calculation, we calculate the frequency dependence of $\operatorname{NOE}(\omega, n, m)$ by using Eqs. (18) and (23), and the parameters from Tables I and II. The frequency dependence of NOE for dendrimers with $n=2-5$ generations is shown in Fig. 9. In the high-frequency region, $\operatorname{NOE}(\omega, n, m)$, as well as $1 / T_{1 \mathrm{C}}^{*}$ are determined by $\tau_{\mathrm{av}}^{\mathrm{int}}$ only. In this limit $\operatorname{NOE}(\omega, n, m)$ does not depend on $n$ and $m$. In the low-frequency region $\operatorname{NOE}(\omega, n, m)$ depends on the size of a marked subbranch and on the size of a dendrimer, i.e., on both values of $n$ and $m$. For $m=0,1$ the $\operatorname{NOE}(\omega, n, m)$ is determined only by $\tau_{m}^{\text {pul }}$ and does not practically depend on $\tau_{\text {rot }}(n)$. $\operatorname{NOE}(\omega, n, m)$ depends on $n$ for $m=2$. The characteristic frequency $\omega_{\mathrm{ch}}$ of the onset of $\operatorname{NOE}(\omega, n, m)$ decrease depends on the maximal relaxation time of $P_{2} \mathrm{ACF}$,

$$
\tau_{333}=\tau_{\text {rot }}(n) / 3 .
$$

The correlation between $\omega_{\mathrm{ch}}$ and $\tau_{\text {rot }}(n)$ is determined by the expression

$$
\omega_{\mathrm{ch}} \approx \frac{1}{4.97}\left(\frac{3}{\tau_{\text {rot }}(n)}\right)
$$

because for low frequencies the maximal contribution to $\operatorname{NOE}(\omega, n, m)$ is equal to $6 J(4.97 \omega)$ [see Eq. (19)]. Note that Eq. (30) and the frequency dependence of $\operatorname{NOE}(\omega, n, m)$ for a dendrimer can be used for the calculation of $\tau_{\text {rot }}(n)$ and for the examination of the developed theory by the comparison of $\tau_{\text {rot }}(n)$ with the results of other experimental methods (for example, dynamic light scattering).
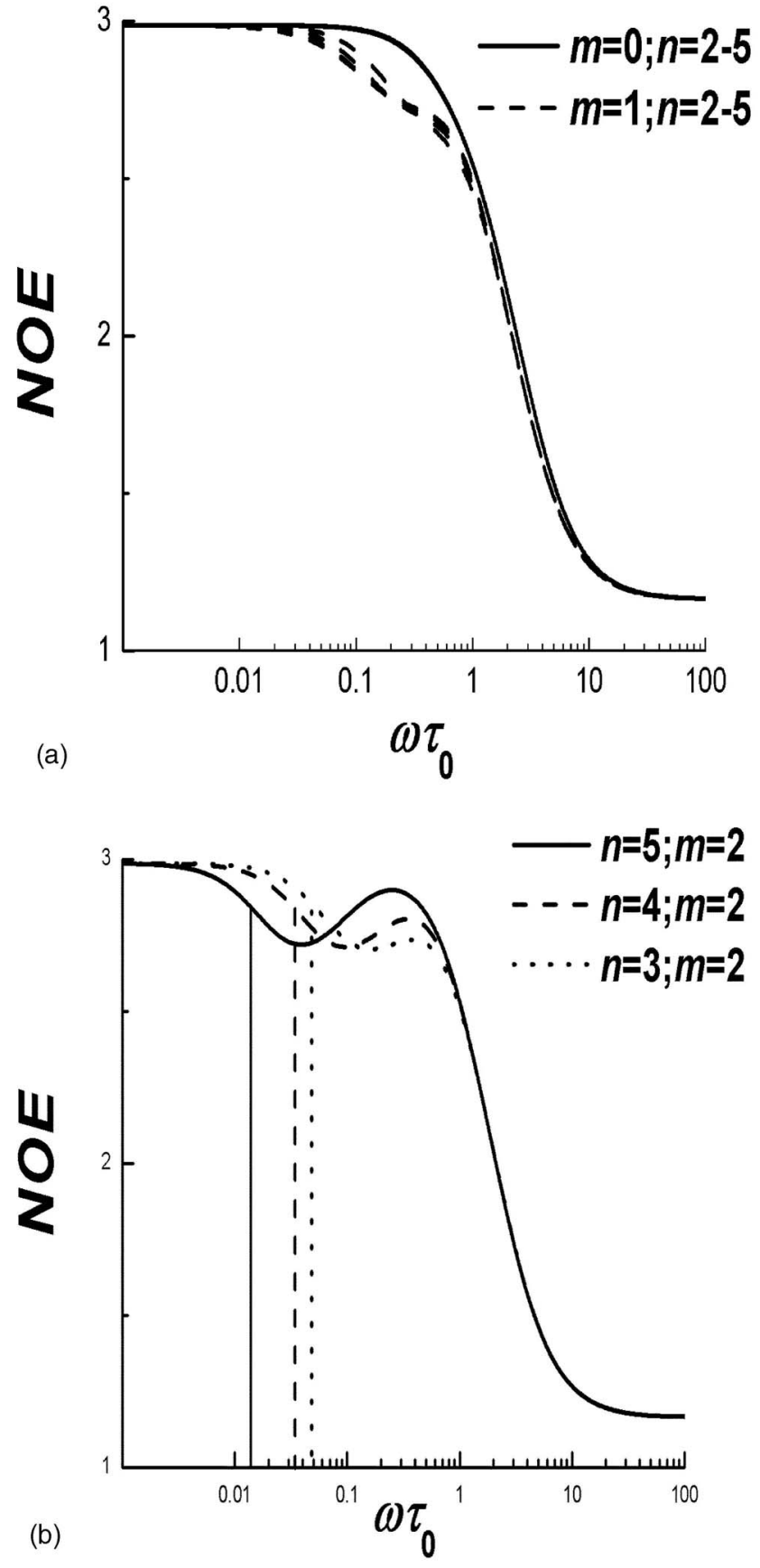

FIG. 9. The frequency dependence of the NOE for the InS of a dendrimer with (a) $m=0,1$ and (b) $m=2$. Vertical lines correspond to the characteristic times NOE in the low-frequency region. $m$ is the number of generations of a marked subbranch, $n$ is the number of dendrimer generations, $\tau_{0}$ is the characteristic relaxation time of a single segment.

Thus, the correlation between the characteristic frequency of the NMR relaxation dependence and the relaxation spectrum of a dendrimer have been found. To the best of our knowledge, there is no experimental data on the frequency dependences of $1 / T_{1}$ and NOE for a dendrimer. We plan to carry out such experiments in the nearest future.

\section{CONCLUSIONS}

In the present study we have tried to improve the analytical approach to calculate the $P_{1}(t) \mathrm{ACF}$ relaxation times 
of a dendrimer by the direct comparison with BD simulation data. Predictions of the developed theory are in a qualitative agreement with the results of the molecular dynamic simulations of a dendrimer in dilute solution. ${ }^{19}$

The relaxation of an orientational $P_{1}(t)$ ACF of an individual dendrimer segment is determined by three processes with rather different characteristic times. The first process corresponds to the local reorientation motions of an InS with the average time of the internal spectrum of the dendrimer. This time does not depend practically on the size of a dendrimer and the topological location of an InS. The second process corresponds to a rotation of a dendrimer branch that originates from this InS. The characteristic time of this process is the maximal pulsating time of the branch and depends only on the number of generations of this branch. The third process is the rotation of a dendrimer as a whole with the characteristic time depending only on the size of a dendrimer. We found that the local orientational mobility of the InS (i.e., not taking into account the rotation of a dendrimer as a whole) depends only on the topological distance between this segment and the terminal shell of a dendrimer.

Improved theoretical approach is also used to calculate analytically the frequency dependence for both NMR relaxation of ${ }^{13} \mathrm{C}$ nuclei and NOE. We established the correlation between the characteristic frequencies of these dependences and the relaxation spectrum of a dendrimer.

\section{ACKNOWLEDGMENTS}

Helpful discussions with Dr. Vladimir A. Shevelev and Dr. Isaak A. Torchinsky are gratefully acknowledged. This work was supported by the INTAS (Grant No. 05-109-4111), the Russian Foundation of Basic Research (Grant Nos. 08-03-00150, 08-03-00565, and 08-03-01139), and The Program of Fundamental Research of CMS Department of Russ. Ac. Sc. - 3 (2009)

${ }^{1}$ J. M. J. Frechet and D. A. Tomalia, Dendrimers and Other Dendritic Polymers (Wiley, New York, 2001).

${ }^{2}$ J. M. J. Frechet, Science 263, 1710 (1994).

${ }^{3}$ S. Svenson and D. A. Tomalia, Adv. Drug Delivery Rev. 57, 2106 (2005)

${ }^{4}$ J. F. Kukowska-Latallo, A. U. Bielinska, J. Johnson, R. Spindler, D. A. Tomalia, and J. R. Baker, Proc. Natl. Acad. Sci. U.S.A. 93, 4897 (1996).

${ }^{5}$ R. Esfand and D. A. Tomalia, Drug Discovery Today 6, 427 (2001).

${ }^{6}$ M. J. Cloninger, Curr. Opin. Chem. Biol. 6, 742 (2002).

${ }^{7}$ C. Dufes, I. F. Uchegbu, and A. G. Schatzlein, Adv. Drug Delivery Rev. 57, 2177 (2005).

${ }^{8}$ S. K. Emran, G. R. Newkome, C. D. Weis, and J. P. Harmon, J. Polym. Sci., Part B: Polym. Phys. 37, 2025 (1999).

${ }^{9}$ A. D. Meltzer, D. A. Tirrel, A. A. Jones, and P. T. Inglefield,
Macromolecules 25, 4541 (1992).

${ }^{10}$ D. Meltzer, D. A. Tirrel, A. A. Jones, and P. T. Inglefield, Macromolecules 25, 4549 (1992).

${ }^{11}$ B. Stark, B. Stuhn, H. Frey, C. Lach, K. Lorenz, and B. Frick, Macromolecules 31, 5415 (1998).

${ }^{12}$ S. Uppuluri, F. A. Morrison, and P. R. Dvornic, Macromolecules 33, 2551 (2000).

${ }^{13}$ J. Mijovic, S. Ristic, and J. Kenny, Macromolecules 40, 5212 (2007).

${ }^{14}$ S. V. Lyulin, A. A. Darinskii, A. V. Lyulin, and M. A. J. Michels, Macromolecules 37, 4676 (2004).

${ }^{15}$ A. V. Lyulin, G. R. Davies, and D. B. Adolf, Macromolecules 33, 3294 (2000).

${ }^{16}$ Z. Y. Chen and C. Cai, Macromolecules 32, 5423 (1999).

${ }^{17}$ F. Ganazzoli, R. La Ferla, and G. Raffaini, Macromolecules 34, 4222 (2001).

${ }^{18}$ M. Murat and G. Grest, Macromolecules 29, 1278 (1996).

${ }^{19}$ K. Karatasos, D. Adolf, and G. R. Davies, J. Chem. Phys. 115, 5310 (2001).

${ }^{20}$ K. Karatasos, Macromolecules 38, 4472 (2005).

${ }^{21}$ K. Karatasos, Macromolecules 39, 4619 (2006).

${ }^{22}$ G. K. Dalakoglou, K. Karatasos, S. V. Lyulin, and A. V. Lyulin, J. Chem. Phys. 127, 214903 (2007).

${ }^{23}$ W. W. Graessley, Macromolecules 13, 372 (1980).

${ }^{24}$ A. Kloczkowski, J. E. Mark, and H. L. Frisch, Macromolecules 23, 3481 (1990).

${ }^{25}$ C. Cai and Z. Y. Chen, Macromolecules 30, 5104 (1997).

${ }^{26}$ P. Biswas, R. Kant, and A. Blumen, Macromol. Theory Simul. 9, 56 (2000).

${ }^{27}$ A. A. Gurtovenko and A. Blumen, Adv. Polym. Sci. 182, 171 (2005).

${ }^{28}$ Yu. Ya. Gotlib and D. A. Markelov, Polym. Sci., Ser. A 44, 1341 (2002).

${ }^{29}$ A. A. Gurtovenko, Yu. Ya. Gotlib, and A. Blumen, Macromolecules 35, $7481(2002)$

${ }^{30}$ A. A. Gurtovenko, D. A. Markelov, Yu. Ya. Gotlib, and A. Blumen, J. Chem. Phys. 119, 7579 (2003).

${ }^{31}$ Yu. Ya. Gotlib and D. A. Markelov, Polymer Sci., Ser. A 46, 815 (2004).

${ }^{32}$ Yu. Ya. Gotlib and D. A. Markelov, Polymer Sci., Ser. A 49, 1137 (2007).

${ }^{33}$ M. Doi and S. R. Edwards, The Theory of Polymer Dynamics (Clarendon, Oxford, 1986).

${ }^{34}$ Yu. Ya. Gotlib and A. I. Neelov, Polymer Sci., Ser. A 45, 1668 (2003).

${ }^{35}$ S. V. Lyulin, L. J. Evers, P. van der Schoot, A. A. Darinskii, A. V. Lyulin, and M. A. J. Michels, Macromolecules 37, 3049 (2004).

${ }^{36}$ E. V. Anufrieva, Yu. Ya. Gotlib, M. G. Krakovjak, I. A. Torchinsky, T. V. Sheveleva, and D.V. Shestopalov, Polym. Sci. U.S.S.R. 15, 2874 (1973).

${ }^{37}$ M. Wind, K. Saalwachter, U.-M. Wiesler, K. Mullen, and H. W. Spiess, Macromolecules 35, 10071 (2002).

${ }^{38}$ X.-Yu. Cao, X.-H. Liu, X.-H. Zhou, Y. Zhang, Y. Jiang, Y. Cao, Yu.-X. Cui, and J. Pei, J. Org. Chem. 69, 6050 (2004).

${ }^{39}$ K. Koynov, G. Mihov, M. Mondeshki, C. Moon, H. W. Spiess, K. Mullen, H.-J. Butt, and G. Floudas, Biomacromolecules 8, 1745 (2007).

${ }^{40}$ D. Doddrell, V. Glushko, and A. Allrhand, J. Chem. Phys. 56, 3683 (1972).

${ }^{41}$ Yu. Ya. Gotlib, I. M. Neelov, I. A. Torchinsky, and V. A. Shevelev, Acta Polym. 40, 643 (1989).

${ }^{42}$ Yu. Ya. Gotlib, I. A. Torchinski, and V. A. Shevelev, Makromol. Chem., Theory Simul. 2, 13 (1993).

${ }^{43}$ Yu. Ya. Gotlib, N. K. Balabaev, A. A. Darinski, and I. M. Neelov, Macromolecules 13, 602 (1980).

${ }^{44}$ C. Malveau, W. E. Baille, X. X. Zhu, and W. T. Ford, J. Polym. Sci., Part B: Polym. Phys. 41, 2969 (2003). 